The Astrophysical Journal, 305:455-466, 1986 June 1

(C) 1986. The American Astronomical Society. All rights reserved. Printed in U.S.A.

\title{
SHAPES AND CORRELATIONS AS OBSERVATIONAL DISCRIMINANTS FOR THE ORIGIN OF DIFFUSE BANDS
}

\author{
G. Chlewicki, ${ }^{1,2}$ G. P. VAn der ZWet, L. J. Van IJZendoorn, And J. M. Greenberg \\ Astrophysics Laboratory, The University of Leiden \\ AND \\ P. P. Alvarez ${ }^{1}$ \\ Instituto de Astrofisica de Canarias \\ Received 1984 December 13; accepted 1985 July 2
}

\begin{abstract}
We have measured the intensities of several diffuse interstellar bands in intermediate resolution spectra $(0.35-0.65 \AA)$ of heavily reddened early-type stars in three associations: Cas OB6, Be 87 , and Cyg OB2. Several independent arguments indicate that none of the stars, with the exception of Cyg OB2 (VI Cyg) No. 12 , is significantly affected by extinction originating in clouds with $n_{\mathbf{H}}>1000 \mathrm{~cm}^{-3}$. The high quality of the data (signal to noise $[\mathrm{S} / \mathrm{N}]$ generally greater than 100 and sometimes as high as 250 ), combined with the strength of the features due to heavy reddening, has made it possible to deduce important new information concerning the properties of the lines. The diffuse lines characterized by sharply peaked profiles $(\lambda 5780, \lambda 5797$, $\lambda 5849, \lambda 6195, \lambda 6203, \lambda 6269, \lambda 6284)$ clearly separate into at least two groups, with $\lambda 5797$ and possibly $\lambda 5849$ being well correlated with $E_{B-V}$, whereas for other lines there is virtually no correlation with extinction within individual associations. Lines for which well-defined profiles could be obtained $(\lambda 5780, \lambda 5797, \lambda 6269, \lambda 6284)$ are all characterized by asymmetric outlines, with the short wavelength wing being the steeper. The degree of asymmetry and details in the outline of the wings are distinctly nonuniform, ranging from a nearly symmetric "Lorentzian" shape for $\lambda 6284$ to a very extended long wavelength wing in $\lambda 6269$. These observed nonuniformities in the properties of the diffuse features appear to make it impossible to explain the origin of the lines as being due to impurity absorption in solid particles. The alternative explanation is to associate the lines with large molecules or exceedingly small amorphous particles $(\lesssim 0.001 \mu \mathrm{m})$ which cannot be described by macroscopic bulk properties.

Subject headings: clusters: associations — interstellar: grains — interstellar: matter
\end{abstract}

\section{INTRODUCTION}

Ever since their interstellar nature was established by Merrill (1934), the diffuse lines have been the subject of intensive observational and theoretical research. In spite of considerable effort expended in their study, the interpretation of the lines remains controversial. Positive correlation of the features with extinction (Herbig 1975; Snow, York, and Welty 1977) has often been taken as an indication that they are associated with absorptions by impurities embedded in interstellar grains. Alternatively, the lines can be explained as electronic or vibronic transitions in molecules, although no specific spectroscopic identification has so far been proposed. Accurate measures of the intensity and profiles of the lines are notoriously difficult, mainly because of uncertainties in setting the continuum level and because of low optical depths of the features in most lines of sight which can be easily observed. Consequently, the exact degree of correlation between the intensities of the lines and the reddening remains uncertain, with strong indications from statistical studies that at least some features correlate with $E_{B-V}$ rather poorly (e.g., Wu, York, and Snow 1981).

The profiles of the lines offer the best insight into the physical mechanism of their formation, but high-resolution data currently available in the literature cover only a few of the

\footnotetext{
${ }^{1}$ Guest Observer at the Observatorio del Roque de los Muchachos.

${ }^{2}$ Reprint requests to G. Chlewicki. Current address: Laboratory for Space Research, and Kapteyn Astronomical Institute, Groningen, The Netherlands.
}

strongest lines and are not sufficient to serve as a definitive constraint on the interpretation of the features. The advent of new, highly sensitive detectors, particularly the CCD, has created an opportunity to resolve some of the outstanding uncertainties in the properties of the lines. In this paper, we present the results of the first observing program in a continuing series, in which diffuse bands are being studied in great detail using high-resolution spectra of heavily reddened stars.

\section{SELECTION OF PROGRAM STARS}

The objects we have selected for our program belong to three stellar aggregates: Cyg OB2, Be 87, and Cas OB6. Since the intensities of the diffuse bands appear to be dependent on the galactic location of the objects (Herbig 1975), two of the groups, Cyg OB2 and Be 87, have been chosen in the same part of the Galaxy (the Cygnus arm), with Cas OB6 (the Perseus arm) used as a control object.

The Cyg OB2 association contains some of the most heavily reddened stars accessible to visual techniques. In particular, one of its members is the extremely luminous supergiant Cyg OB2 No. 12 , reddened by $E(B-V) \approx 3.4 \mathrm{mag}$ (Morgan, Johnson, and Roman 1954; Schulte 1958; Souza and Lutz $1980 a$ ). The association is located at a distance of $\sim 2 \mathrm{kpc}$ from the Sun in the direction of the Cygnus arm $\left(1=80^{\circ}\right)$. Very strong differential reddening with $E(B-V)$ ranging from 1.5 to 3.4 mag indicates that the dust seen in more heavily reddened stars is associated with clouds in the vicinity of the cluster. The extinction curves for Cyg OB2 stars in the visible and IR 
regions of the spectrum are normal, with $R=A_{V} / E_{B-V} \approx 3$ (Johnson 1968). This implies that there are no extra accreted mantles on the large grains responsible for extinction at long wavelengths. The same conclusion is also indicated by the lack of any $\mathrm{H}_{2} \mathrm{O}$ absorption in the line of sight toward Cyg OB2 No. 12 (Voelcker and Elsasser 1973). Typical densities in clouds in the vicinity of Cyg OB2 can be estimated from the differential reddening within the group. Assuming a normal gas-to-dust ratio and taking into account the clumpiness of the interstellar medium, one arrives at $n_{\mathrm{H}}$ from $\sim 100 \mathrm{~cm}^{-3}$ to $\sim 500 \mathrm{~cm}^{-3}$ as a characteristic density of clouds contributing most of the extinction. Only star No. 12 can be substantially affected by extinction arising in dense clouds with $n_{\mathrm{H}} \gtrsim 10^{3}$ $\mathrm{cm}^{-3}$. Some $\mathrm{CO}$ emission has been observed in the direction of Cyg OB2 with a velocity consistent with that of $\mathrm{C}_{2}$ lines in star No. 12 (Souza and Lutz 1980b). The set of properties we have outlined defines the Cyg OB2 cloud as an intermediate case between molecular clouds as observed at radio wavelengths and the general diffuse medium. Two of the stars (No. 10 and No. 12) have been known to exhibit probably the strongest diffuse features ever observed at 5780 and $6284 \AA$ (Bromage and Nandy 1973).

The relatively nearby group Be 87 lies at a distance of $0.95 \mathrm{kpc}$ from the Sun and forms part of a complex of molecular clouds in the Cygnus $\mathrm{X}$ region which contains several young associations. In their recent paper, Turner and Forbes (1982) have presented photometric observations of $\mathrm{Be} 87$ and traced the edge of a large molecular cloud across the cluster by mapping the extinction observed for individual stars. The four stars we have chosen have increasing reddening, and their locations within the cluster (see Fig. 6 in Turner and Forbes 1982) indicate that, moving from No. 7 to No. 18, the line of sight contains increasing amounts of material belonging to the molecular cloud observed by Turner and Forbes. Although there is no ongoing star formation in the cluster, it appears to be associated with nearby star formation indicators (H II regions, $\mathrm{H}_{2} \mathrm{O}$ masers; see Pitault 1981). Furthermore, distance estimates for the molecular cloud complex based on observations of $\mathrm{H}$ II regions (Eiroa, Elsasser, and Lahulla 1978) confirm the picture which places the Be 87 stars immediately behind the fringe of a large molecular cloud. There are no observations of IR extinction toward $\mathrm{Be} 87$, therefore it is not possible to decide whether substantial accretion on large grains may be taking place in the cloud. A typical cloud density derived from differential reddening is $n_{\mathrm{H}} \approx 300 \mathrm{~cm}^{-3}$, which implies that, although some of the most heavily reddened stars may be seen through clouds with $n_{\mathrm{H}} \approx 10^{3} \mathrm{~cm}^{-3}$, most of the material is contained in clouds with densities similar to those encountered in Cyg OB2. We shall demonstrate in the following section that this is consistent with observations of diffuse lines.

The Cas OB6 association, with the IC 1805 open cluster as its core, is located in the Perseus arm $(d \approx 2 \mathrm{kpc})$. It was cited by Lada et al. (1978) as an example of sequential star formation. The group is interacting with a major molecular cloud seen in CO maps, although within the association itself there is little or no molecular material. Observations in the UV have revealed that for all Cas OB6 stars observed in this program, there are no peculiarities in the UV extinction curve. This strongly suggests normal diffuse medium properties for all major components of the interstellar grain population (Greenberg and Chlewicki 1983; Chlewicki et al. 1984). No deviations from the average extinction are indicated in the IR
(Johnson 1968) confirming normal sizes of large grains. Because the extinction to the Cas OB6 stars best exhibits the characteristics of normal diffuse medium grains, this association has been chosen as a source of control objects in which the interstellar features might be expected to follow the pattern usually observed for diffuse medium dust.

\section{OBSERVATIONS}

The observations were carried out during three consecutive nights, 1984 July 7-9 at the Observatorio del Roque de los Muchachos using the RGO intermediate dispersion spectrograph on the $2.5 \mathrm{~m}$ Isaac Newton telescope. The spectrograph was used in its highest resolution mode, with a $500 \mathrm{~mm}$ camera and an 1800 grooves per $\mathrm{mm}$ holographic grating, producing a dispersion of $\sim 10 \AA$ per $\mathrm{mm}$ in the spectral range observed. The detector was a GEC CCD array with a pixel size of $22 \mu \mathrm{m}$ corresponding to the maximum spectral resolution of $0.22 \AA$. All engineering data for the intensity and wavelength calibration were obtained independently on each night. The detector was preflashed in order to increase sensitivity. The background produced by preflashing was calibrated and subtracted from every image. Tungsten lamp flat-field images were used to calibrate the pixel-to-pixel response of the detector; the final response map was based on the average of six exposures. The wavelength calibration was derived from copper-argon lamp exposures and was checked by measuring the positions of interstellar and stellar lines in the spectra.

In order to increase the efficiency, the slit widths used in the observations were larger than the nominal setting required to achieve the maximum resolution of $0.22 \AA$. As a result, the effective resolution was $0.35 \AA$ for the stars in Cyg OB2 and Cas OB6, and $0.66 \AA$ for most objects in Be 87 (other stars in the group were observed at higher resolution). The quality of the spectra was further improved by averaging the signal in the direction of dispersion in bins of either 2 or 3 pixels, depending on the maximum resolution determined by the slit. Spikes caused by the impact of cosmic rays were removed by interpolation between the nearest pixels outside the area, usually with a diameter of 3-4 pixels, which was affected by the particle event.

The stars in Cyg OB2 and Be 87 were observed in two wavelength windows: $5765-5890 \AA$ and $6170-6300 \AA$. The objects in Cas OB6 were observed only in the short wavelength window. The spectra in the $6300 \AA$ window required an additional correction to remove the telluric $\mathrm{O}_{2}$ lines, with a band head at $6276 \AA$, which severely distort the profile of $\lambda 6284$. The optical depth of telluric absorptions, $\tau_{\mathrm{O}_{2}}(\lambda)$, was derived from a trailed spectrum of $\eta \mathrm{UMa}\left(S_{p}=\mathrm{B} 3 \mathrm{~V}, V=1.8 \mathrm{mag}\right.$, $E_{B-V}=0.01 \mathrm{mag}$ ) which was taken with a signal-to-noise ratio of $\sim 1000$. Each program spectrum was divided by $\exp \left[-C \tau_{\mathrm{O}_{2}}(\lambda)\right]$, where the factor $C$, which accounts for the different air mass in each line of sight, was varied until the best cancellation of the $\mathrm{O}_{2}$ lines was achieved. Although the resolution was not sufficient to remove the telluric lines entirely, residual $\mathrm{O}_{2}$ absorption appears only as increased noise in the wings of $\lambda 6284$ and does not preclude the determination of the profile.

The relevant data for all spectra are shown in Table 1. Signal-to-noise values given there are based on estimates of the sensitivity of the detector and its figure of merit obtained during the observations. The numbers quoted in parentheses are $\mathrm{S} / \mathrm{N}$ ratios directly measured in the featureless, central portion of each spectrum (Fig. 1). 


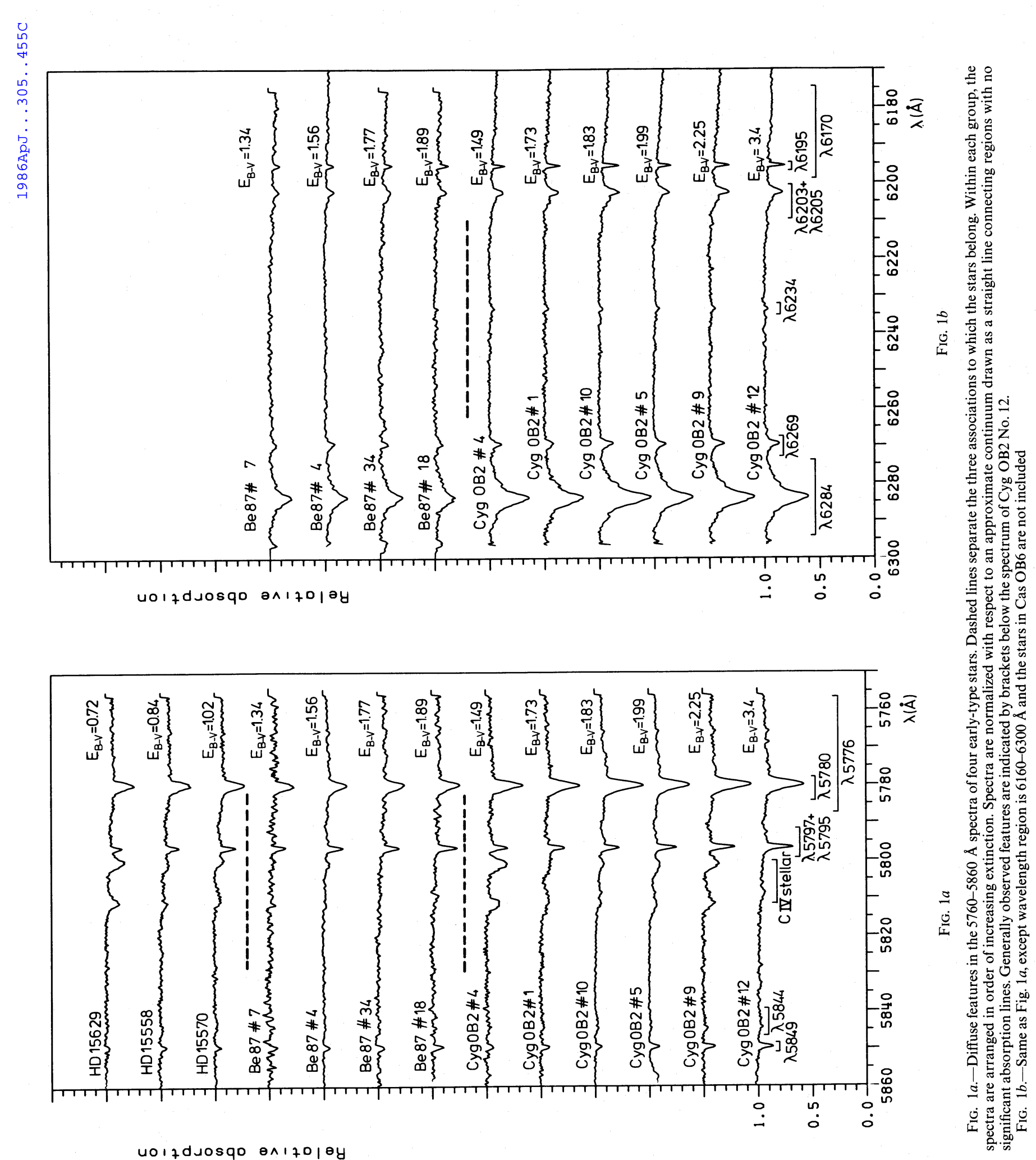


TABLE 1

ObServational Parameters of Program Stars

\begin{tabular}{|c|c|c|c|c|c|c|c|}
\hline Star & $\begin{array}{l}\text { Spectral Type/ } \\
\text { Luminosity Class }\end{array}$ & $E(B-V)$ & V & $\begin{array}{c}\text { Wavelength } \\
\text { Window } \\
(\AA)\end{array}$ & $\begin{array}{l}\text { Integration } \\
\text { Time } \\
\text { (s) }\end{array}$ & $\begin{array}{l}\text { Slit } \\
\text { Width } \\
(\mu \mathrm{m})\end{array}$ & $\mathrm{S} / \mathrm{N}^{\mathrm{b}}$ \\
\hline $\begin{array}{l}\mathrm{HD} 15629 \ldots \ldots \ldots \ldots \\
\quad\left(\mathrm{BD}+60^{\circ} 507, \text { Cas OB } 6\right)\end{array}$ & $\mathrm{O} 5 \mathrm{~V}$ & 0.72 & 8.43 & 5800 & 200 & 100 & $110(110)^{\mathrm{c}}$ \\
\hline $\begin{array}{l}\text { HD } 15558 \ldots \ldots \ldots \ldots \\
\quad\left(\mathrm{BD}+60^{\circ} 502, \text { Cas OB } 6\right)\end{array}$ & O5 III & 0.84 & 7.81 & 5800 & 100 & 100 & $90(95)$ \\
\hline $\begin{array}{l}\text { HD } 15570 \ldots \ldots \ldots \ldots \ldots \\
\quad\left(\mathrm{BD}+60^{\circ} 504, \text { Cas OB } 6\right)\end{array}$ & O4 If & 1.02 & 8.10 & 5800 & 140 & 100 & $100(110)$ \\
\hline Be 87 No. $7 \ldots \ldots \ldots \ldots$ & $B^{d}$ & 1.34 & 13.02 & $\begin{array}{l}5800 \\
6200\end{array}$ & $\begin{array}{l}2400 \\
3600\end{array}$ & $\begin{array}{l}100 \\
200\end{array}$ & $\begin{array}{r}50(45) \\
105(95)\end{array}$ \\
\hline Be 87 No. $4 \ldots \ldots$ & $\mathrm{O}^{\mathrm{d}}$ & 1.56 & 10.92 & $\begin{array}{l}5800 \\
6200\end{array}$ & $\begin{array}{l}1850 \\
1700\end{array}$ & $\begin{array}{l}100 \\
100\end{array}$ & $\begin{array}{l}150(155) \\
150(160)\end{array}$ \\
\hline Be 87 No. $34 \ldots \ldots \ldots \ldots$ & $\mathrm{B}^{\mathrm{d}}$ & 1.77 & 13.32 & $\begin{array}{l}5800 \\
6200\end{array}$ & $\begin{array}{l}4200 \\
4200\end{array}$ & $\begin{array}{l}200 \\
200\end{array}$ & $\begin{array}{l}80(75) \\
90(75)\end{array}$ \\
\hline Be 87 No. $18 \ldots .$. & $B^{d}$ & 1.89 & 12.84 & $\begin{array}{l}5800 \\
6200\end{array}$ & $\begin{array}{l}2700 \\
3000\end{array}$ & $\begin{array}{l}100 \\
200\end{array}$ & $\begin{array}{r}70(60) \\
100(85)\end{array}$ \\
\hline $\begin{array}{l}\text { Cyg OB2 No. } 4 \ldots \\
\left.\text { (BD }+40^{\circ} 4219\right)\end{array}$ & O7 III & 1.49 & 10.22 & $\begin{array}{l}5800 \\
6200\end{array}$ & $\begin{array}{l}550 \\
900\end{array}$ & $\begin{array}{l}100 \\
100\end{array}$ & $\begin{array}{c}80(90) \\
130(140)\end{array}$ \\
\hline Cyg OB2 No. $1 \ldots \ldots \ldots \ldots$ & $\mathrm{O} 9 \mathrm{~V}$ & 1.73 & 11.09 & $\begin{array}{l}5800 \\
6200\end{array}$ & $\begin{array}{l}1200 \\
1400\end{array}$ & $\begin{array}{l}100 \\
100\end{array}$ & $\begin{array}{r}80(90) \\
100(95)\end{array}$ \\
\hline $\begin{array}{l}\text { Cyg OB2 No. } 10 \ldots \ldots \ldots \ldots \\
\quad\left(\mathrm{BD}+41^{\circ} 3804\right)\end{array}$ & O9.5 Ia & 1.83 & 9.87 & $\begin{array}{l}5800 \\
6200\end{array}$ & $\begin{array}{r}3000 \\
600\end{array}$ & $\begin{array}{l}100 \\
100\end{array}$ & $\begin{array}{l}180(175) \\
130(130)\end{array}$ \\
\hline $\begin{array}{l}\text { Cyg OB2 No. } 5^{\mathrm{e}} \\
\quad\left(\mathrm{BD}+40^{\circ} 4220\right)\end{array}$ & O7 Ia & 1.99 & $9.10 \mathrm{v}$ & $\begin{array}{l}5800 \\
6200\end{array}$ & $\begin{array}{r}1200 \\
600\end{array}$ & $\begin{array}{l}100 \\
100\end{array}$ & $\begin{array}{l}240(245) \\
180(150)\end{array}$ \\
\hline Cyg OB2 No. $9 \ldots \ldots \ldots \ldots$ & O5 If & 2.25 & 10.80 & $\begin{array}{l}5800 \\
6200\end{array}$ & $\begin{array}{r}900 \\
1100\end{array}$ & $\begin{array}{l}100 \\
100\end{array}$ & $\begin{array}{r}90(100) \\
130(110)\end{array}$ \\
\hline Cyg OB2 No. $12 \ldots \ldots \ldots$ & $\mathrm{B} 8 \mathrm{Ia}^{\mathrm{f}}$ & $\sim 3.4$ & 11.5 & $\begin{array}{l}5800 \\
6200\end{array}$ & $\begin{array}{l}2700 \\
2700\end{array}$ & $\begin{array}{l}100 \\
100\end{array}$ & $\begin{array}{l}110(105) \\
190(155)\end{array}$ \\
\hline
\end{tabular}

a $5800 \AA$ refers to the wavelength range extending from 5670 to $5885 \AA$, and $6200 \AA$ denotes the wavelength window from 6170 to $6300 \AA$.

${ }^{b}$ Calculated for binned spectra (see text).

c Signal-to-noise radio directly measured in the spectrum.

d Based on the photometry given by Turner and Forbes 1982 and on spectral characteristics observed in this program.

e Binary star, photometrically variable and with peculiar spectroscopic characteristics.

${ }^{\mathrm{f}}$ Estimates of spectral class vary within about five subclasses with corresponding uncertainty in $E(B-V)$ of $0.1-0.2 \mathrm{mag}$.

\section{RESULTS}

All spectra obtained in this program are shown in Figures $1 a$ and $1 b$. Detailed analysis of the equivalent widths of individual lines will be described below, but several conclusions can be drawn on the basis of Figure 1 alone. Perhaps the most striking is the behavior of strong lines such as $\lambda 5780$ and $\lambda 6284$ in Be 87 and Cyg OB2. The two lines are significantly weaker in all stars in $\mathrm{Be} 87$, even though the reddening is similar to, or even higher than, that of several stars in Cyg OB2. The intensities of the lines do not show any dependence on $E_{B-V}$ within each association: in $\mathrm{Be} 87$, both lines have an approximately constant intensity, whereas the stars in Cyg OB2 can be roughly divided into two groups with, respectively, weak (No. 4, No. 1, No. 5) and strong (No. 10, No. 9, No. 12) diffuse lines. This erratic pattern is followed by most lines whose sharp profiles allow accurate determination of intensity ( $\lambda 6269$, $\lambda 6203$, 26195). The most notable exception is $\lambda 5797$, which consistently increases with the reddening in all stars in our program. The narrow feature at $5849 \AA$ is too weak in most spectra to permit measurements of its intensity but on the basis of the spectra of the most heavily reddened stars in Cyg OB2, it seems to follow the behavior of 25797 . This preliminary analysis of the data therefore suggests that the diffuse lines in the two wavelength windows we have observed can be divided into two groups, with $\lambda 5797$ and, probably, $\lambda 5849$ (group I) being strongly dependent on $E_{B-V}$, and $\lambda 5780, \lambda 6195, \lambda 6203$, $\lambda 6269$, and $\lambda 6284$ (group II) showing hardly any such dependence.

Table 2 contains the equivalent widths for those lines for which they can be measured in all program stars. The equivalent width given for $\lambda 6203$ includes all features in the highly complex, multiple structure between $\sim 6200 \AA$ and $\sim 6207 \AA$. The blended, double profile of $\lambda 5797+\lambda 5795$ also requires a common continuum for both lines, but the outlines of individual features are sufficiently clear for the equivalent width of $\lambda 5797$ to be measured separately. The errors in the equivalent width are dominated by the uncertainties in setting the continuum. Accurate numerical estimates of the magnitude of the errors are therefore difficult. The values quoted in Table 2 have been derived separately for each association as rms deviations from the average width in profiles normalized to the central absorption of $A_{c}=0.5$. The estimates have been raised when other sources of errors appeared to be involved; e.g., insufficient resolution for $\lambda 6195$. The presence of stellar lines significantly affects only the $5797 \AA$ feature, which in O stars is blended with the stellar C IV at $\sim 5801 \AA$. In Cyg OB2 No. 12, because of its relatively late spectral type, $\lambda 6269$, may be blended with the neighboring Ne I line at $6266 \AA$. In general, 
TABLE 2

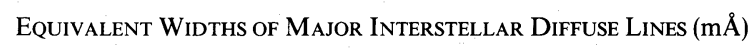

\begin{tabular}{|c|c|c|c|c|c|c|c|c|c|c|c|c|c|}
\hline Object & $E(B-V)$ & $W_{5797}$ & $\epsilon^{a}$ & $W_{5780}$ & $\epsilon^{\mathrm{a}}$ & $W_{6195}$ & $\epsilon^{\mathrm{a}}$ & $W_{6203}$ & $\epsilon^{a}$ & $W_{6269}$ & $\epsilon^{\mathrm{a}}$ & $W_{6284}$ & $\epsilon^{\mathrm{a}}$ \\
\hline $\begin{array}{l}\text { HD } 15269 \ldots \ldots \ldots \ldots \\
\quad \text { (Cas OB6) }\end{array}$ & 0.72 & 92.7 & \multirow{3}{*}{0.13} & 470.7 & & $\ldots$ & $\ldots$ & $\ldots$ & $\ldots$ & $\ldots$ & $\ldots$ & $\ldots$ & .. \\
\hline $\begin{array}{l}\text { HD } 15558 \ldots \ldots \ldots \ldots \\
\quad \text { (Cas OB6) }\end{array}$ & 0.84 & 145.1 & & 478.7 & & $\ldots$ & $\ldots$ & $\ldots$ & $\ldots$ & $\ldots$ & $\ldots$ & $\ldots$ & $\ldots$ \\
\hline $\begin{array}{l}\text { HD } 15570 \ldots \ldots \ldots \ldots \\
\quad(\text { Cas OB6) }\end{array}$ & 1.02 & 154.7 & & 466.5 & & $\ldots$ & $\ldots$ & $\ldots$ & $\ldots$ & $\ldots$ & $\ldots$ & $\ldots$ & $\ldots$ \\
\hline $\begin{array}{l}\text { Be } 87 \text { No. } 7 \ldots \ldots \ldots \ldots \\
\text { Be } 87 \text { No. } 4 \ldots \ldots \ldots \ldots \\
\text { Be } 87 \text { No. } 34 \ldots \ldots \ldots \\
\text { Be } 87 \text { No. } 18 \ldots \ldots \ldots\end{array}$ & $\begin{array}{l}1.34 \\
1.56 \\
1.77 \\
1.89\end{array}$ & $\begin{array}{l}169.0 \\
194.2 \\
212.1 \\
229.9\end{array}$ & 0.05 & $\begin{array}{l}494.8 \\
421.2 \\
531.2 \\
506.8\end{array}$ & 0.06 & $\begin{array}{l}60.7 \\
49.8 \\
52.8 \\
69.7\end{array}$ & 0.16 & $\begin{array}{l}287.6 \\
204.5 \\
406.9 \\
294.3\end{array}$ & 0.26 & $\begin{array}{l}112.9 \\
153.7 \\
133.6 \\
166.1\end{array}$ & 0.13 & $\begin{array}{r}1098.8 \\
1017.3 \\
1079.0 \\
943.8\end{array}$ & 0.05 \\
\hline $\begin{array}{l}\text { Cyg OB2 No. } 4 \ldots \ldots \\
\text { Cyg OB2 No. } 1 \ldots \ldots .\end{array}$ & $\begin{array}{l}1.49 \\
1.73\end{array}$ & $\begin{array}{l}192.9 \\
187.0\end{array}$ & \multirow{4}{*}{0.05} & $\begin{array}{l}753.4 \\
775.9\end{array}$ & \multirow{4}{*}{0.02} & $\begin{array}{l}57.3 \\
73.1\end{array}$ & \multirow{4}{*}{0.11} & $\begin{array}{l}375.9 \\
414.8\end{array}$ & \multirow{4}{*}{0.04} & $\begin{array}{l}183.8 \\
149.3\end{array}$ & \multirow{4}{*}{0.05} & $\begin{array}{l}1942.6 \\
1922.2\end{array}$ & \multirow{4}{*}{0.05} \\
\hline Cyg OB2 No. $10 \ldots \ldots$ & $\begin{array}{l}1.83 \\
1.99\end{array}$ & $\begin{array}{l}220.0 \\
240.4\end{array}$ & & $\begin{array}{r}1021.4 \\
787.4\end{array}$ & & $\begin{array}{r}109.3 \\
657\end{array}$ & & 613.2 & & 230.3 & & 2673.2 & \\
\hline $\begin{array}{l}\text { Cyg OB2 No. } 5 \ldots \ldots \ldots \\
\text { Cyg OB2 No. } 9 \ldots \ldots \ldots\end{array}$ & $\begin{array}{l}1.99 \\
2.25\end{array}$ & $\begin{array}{l}240.4 \\
275.7\end{array}$ & & $\begin{array}{r}187.4 \\
1004.3\end{array}$ & & $\begin{array}{l}65.7 \\
80.8\end{array}$ & & $\begin{array}{l}413.9 \\
571.0\end{array}$ & & $\begin{array}{l}166.7 \\
205.2\end{array}$ & & $\begin{array}{l}2047.3 \\
2269.0\end{array}$ & \\
\hline Cyg OB2 No. 12 & 3.4 & 338.4 & & 929.1 & & 87.3 & & 505.1 & & 222.7 & & 2194.0 & \\
\hline
\end{tabular}

${ }^{a} \epsilon$ is the value of the relative error derived from the standard deviation in the equivalent width of normalized profiles.

weak stellar lines totally hidden by broad diffuse bands such as $\lambda 5780$ or $\lambda 6284$ will tend to be included, at least partly, in our estimate of random errors because of the wide range of $\mathrm{O}$ and B spectral types observed in Cyg OB2 and Be 87.

Three lines covered by our observations, $\lambda 5849, \lambda 5844$, and $\lambda 6234$, have not been included in Table 2 . For $\lambda 5849$, the equivalent width can be calculated in only five stars and the results are discussed separately in connection with Table 3 . The 5844 $\AA$ line is too shallow to provide a reliable estimate of the equivalent width. In Be 87 and Cyg OB2, it appears to follow the behavior of the $\lambda 5780$ group of lines (group II), but it is surprisingly strong in HD 15570 , in which $\lambda 5780$ is rather weak. For 26234 , which was classified by Herbig (1975) as " possibly interstellar," our observations confirm that it should be regarded as one of the diffuse bands. The feature is too weak for any quantitative analysis. However, on the basis of the high $\mathrm{S} / \mathrm{N}$ spectrum of Be 87 No. 4, it appears to be weaker in Be 87 than in Cyg OB2, possibly indicating its correlation with group II.

In Figures 2, 3, and 4 we have presented the measures of the equivalent width of the lines in the form of correlation diagrams for those features for which low magnitude of errors $(\lesssim 5 \%)$ justifies such presentation. Figure 2 compares $\lambda 5797$ with $E(B-V)$ and demonstrates a very good correlation for the Cygnus stars, with the correlation coefficient $r$ equal to 0.96 if No. 12 is excluded. In Cyg OB2 No. 12, the line is weaker by an amount which exceeds the likely error at least 3 times. This "saturation" effect resembles similar observations of other lines in molecular clouds (Snow and Cohen 1974). The stars in Cas OB6 do not follow the relation defined by the Cygnus

TABLE 3

Correlation between the Equivalent Widths of Major Diffuse Lines

\begin{tabular}{|c|c|c|c|c|c|c|}
\hline \multirow{2}{*}{$\begin{array}{c}\text { LINE } \\
\text { OBSERVED }\end{array}$} & \multicolumn{6}{|c|}{ CORRElation COEFFicient } \\
\hline & $W_{5797}$ & $W_{5780}$ & $W_{6195}$ & $W_{6203}$ & $W_{6269}$ & $W_{6284}$ \\
\hline$E(B-V)$ & 0.97 & 0.54 & 0.48 & 0.51 & 0.64 & 0.44 \\
\hline$W_{5797} \ldots \ldots \ldots \ldots$ & & 0.58 & 0.48 & 0.55 & 0.69 & 0.46 \\
\hline$W_{5780} \cdots \ldots \ldots \ldots$ & & & 0.81 & 0.94 & 0.83 & 0.97 \\
\hline$W_{6195} \ldots \ldots \ldots$ & & & & 0.82 & 0.79 & 0.78 \\
\hline$W_{6203} \ldots \ldots \ldots$ & & & & & 0.76 & 0.88 \\
\hline$W_{6269} \ldots \ldots \ldots$ & & & & & & 0.81 \\
\hline
\end{tabular}

stars, but no exact calculations are possible since two of the three spectra observed for the group have $\lambda 5797$ badly affected by blending with the stellar C IV at $5801 \AA$. These stars have not been included in the analysis of correlation because earlier research suggested that the intensities of diffuse lines may be systematically different in the Perseus arm and in Cygnus (Herbig 1975). The errors in $E_{B-V}$ have not been shown explicitly in Figure 2 since their low magnitude $(\sim 0.02 \mathrm{mag}$ in Cyg OB2 increasing to $\sim 0.05 \mathrm{mag}$ in Be 87 , where $E_{B-V}$ has been derived from main-sequence fitting) makes them relatively insignificant in these heavily reddened stars. However,

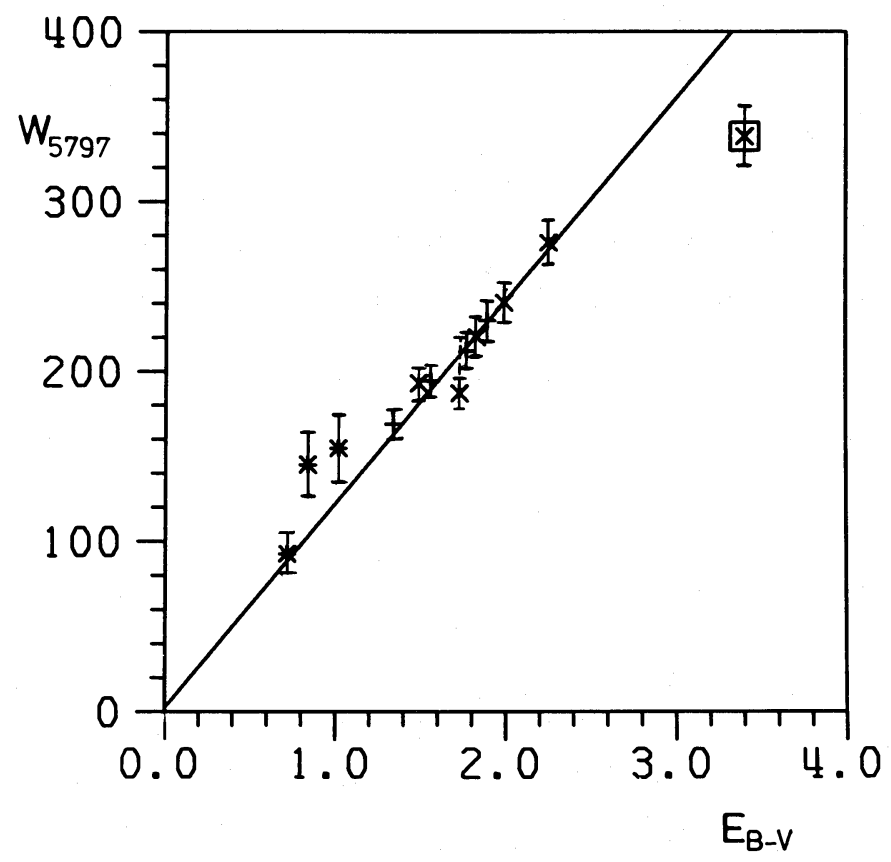

Fig. 2.-Correlation of the equivalent width of $\lambda 5797$ with $E_{B-V}$. Crosses $(\times)$ denote Cyg OB2, plusses (+) Be 87, and asterisks $\left({ }^{*}\right)$ Cas OB6. The regression line is calculated for all stars in Be 87 and Cyg OB2, with the exception of Cyg OB2 No. 12, whose position in the diagram is enclosed by a square. The correlation coefficient is 0.96 . Objects in Cas OB6 are not included (see text). Dashed error bar for Cyg OB2 No. 5 shows the maximum uncertainty due to stellar C IV. 


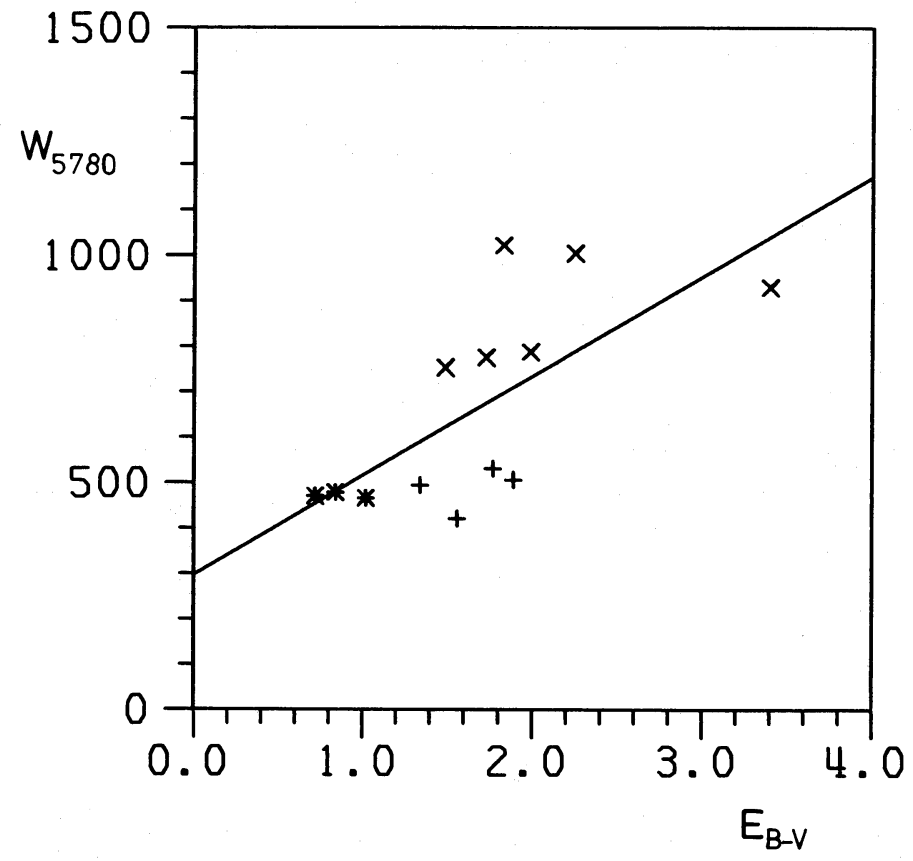

FIG. 3

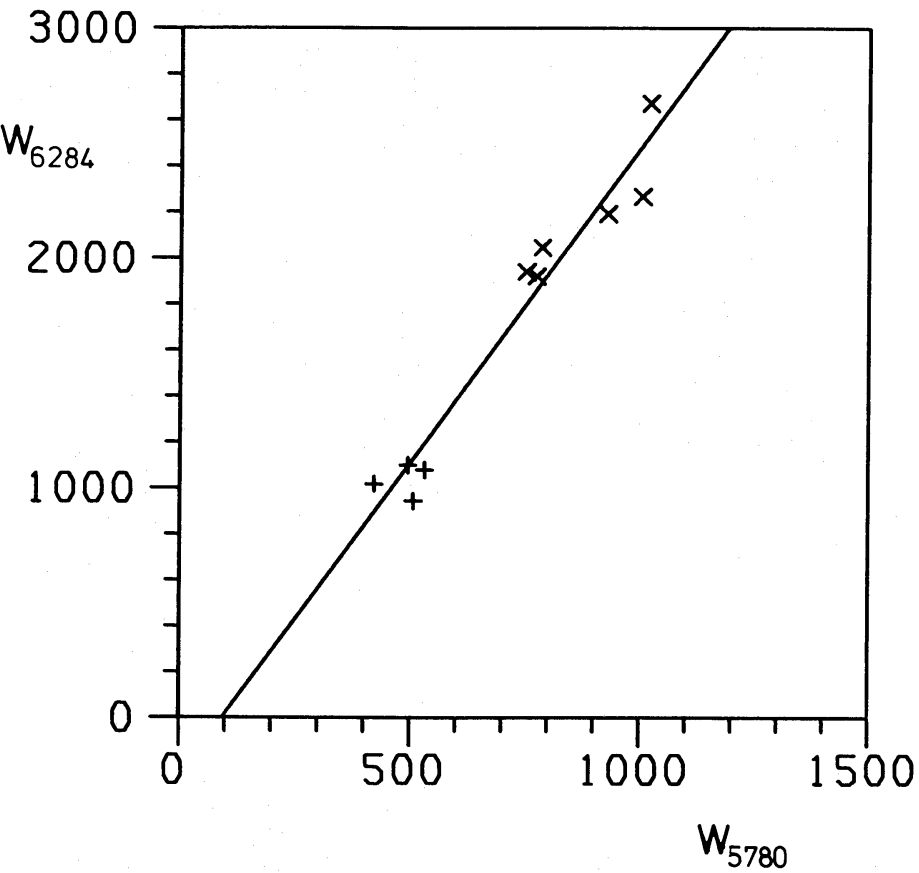

FIG. 4

FIG. 3. - Correlation of the equivalent width of $\lambda 5780$ with $E_{B-V}$. Crosses $(\times)$ denote Cyg OB2, plusses $(+)$ Be 87, and asterisks $\left({ }^{*}\right)$ Cas OB6. The regression line is computed for all points and gives a correlation coefficient of 0.68 .

Fig. 4. - Correlation between the equivalent widths of $\lambda 6284$, and $\lambda 5780$. Crosses $(\times)$ denote Cyg OB2, plusses $(+)$ Be 87 . The regression line is calculated for all objects in the diagram. Correlation coefficient is 0.94 .

two special cases deserve some attention. The classification given for Cyg OB2 No. 12 range from B3 Ia (Abbott, Bieging, and Churchwell 1981) to B8 Ia (Souza and Lutz 1980a), and the corresponding uncertainty in $E_{B-V}$ can be as large as $0.2 \mathrm{mag}$, although the relative error is still only $\sim 5 \%$. Star No. 5 in Cyg OB2 is an eclipsing binary with a $V$-magnitude variability exceeding $0.3 \mathrm{mag}$. However, since both components are O stars (Bohannan and Conti 1976), the $B-V$ of the system hardly changes with phase. The maximum $B-V$ variation we have derived from Hall's (1974) light curves of Cyg OB2 No. 5 is $\sim 0.01 \mathrm{mag}$, so that the error in $E_{B-V}$ is dominated, as for other $\mathrm{O}$ stars, by the uncertainty in the intrinsic color.

Figure 3 presents the data for the $5780 \AA$ line versus $E(B-V)$. The error bars are generally comparable with the size of the symbols and therefore are not shown. Relatively poor correlation described earlier in reference to Figure 1 is again very clearly demonstrated. Figure 4 contains the correlation diagram for $\lambda 6284$ versus $\lambda 5780$. The correlation is convincing $(r=0.94)$, bearing in mind that the errors in the measures of $\lambda 6284$ are increased by telluric $\mathrm{O}_{2}$. As in Figure 3, the error bars have not been shown because they are of the size of the symbols, except in some of the Cyg OB2 stars. The line $\lambda 6284$ may be affected by relatively high systematic errors caused by the large width of the line and residual $\mathrm{O}_{2}$.

The correlation coefficients, calculated without including errors in equivalent widths and $E_{B-V}$, have been collected in Table 3 for all lines observed in Cyg OB2 and Be 87. The explicit introduction of formal errors in correlating variables leads to no significant change in correlation coefficients. Sampling errors are $\sim 0.1$ for low correlation coefficients and become as low as $\sim 0.01$ for $r=0.96$. In general, the data justify the division of all lines into two groups since only $\lambda 5797$ ( $\lambda 5849$ could not be measured in all stars) correlates with
$E(B-V)$ and all other lines in the table correlate well with each other. The errors are large for some of the weaker lines, so the data in Table 3 cannot be taken as conclusive evidence that all lines in group II are components of a single spectrum. The equivalent width of $\lambda 5849$ could be obtained only for the four most heavily reddened stars in Cyg OB2 and Be 87 No. 4, with a typical error of $\sim 15 \%$. Within this relatively large error margin, the ratio $W_{5849} / W_{5797}$ remains nearly constant, whereas $W_{5849} / W_{5780}$ varies by more than a factor of 2 . The $5849 \AA$ feature is therefore likely to belong to the $\lambda 5797$ group of lines (group I). This conclusion, however, rests heavily on the measurements of $W_{5849}$ in Cyg OB2 No. 10 and Be 87 No. 4, in which the lines are rather weak, and further confirmation is required.

There is a weak indication in the numbers contained in Table 3 that $\lambda 6269$ may not belong to either group. The correlation of $W_{6269}$ with $E_{B-V}$ is intermediate between the two groups and so is its correlation with $W_{5780}$ and $W_{6284}$. The line is among the strongest features and the errors in its equivalent width are small, particulary in Cyg OB2, so that the discrepancies are unlikely to be due to poor measurements. However, because of the large sampling error, this result must be treated as only marginally significant.

The resolution of our spectra allowed accurate quantitative determination of the profiles of five lines: $\lambda 5780, \lambda 6284$, and, in some stars, $\lambda 5797, \lambda 6269$, and $\lambda 6203$. The $\lambda 5780$ profile (Fig. $5 a$ ) confirms the asymmetry observed in other studies (Wu 1972; Savage 1976; Danks and Lambert 1976; Snell and Vanden Bout 1981). Profile variations reported by some authors are not apparent in our spectra. The profile of $\lambda 5780$ seems to be identical for all stars within one association after the line has been normalized to the same central absorption of $A_{c}=0.5$; the slight broadening seen in the Be 87 average is an instru- 

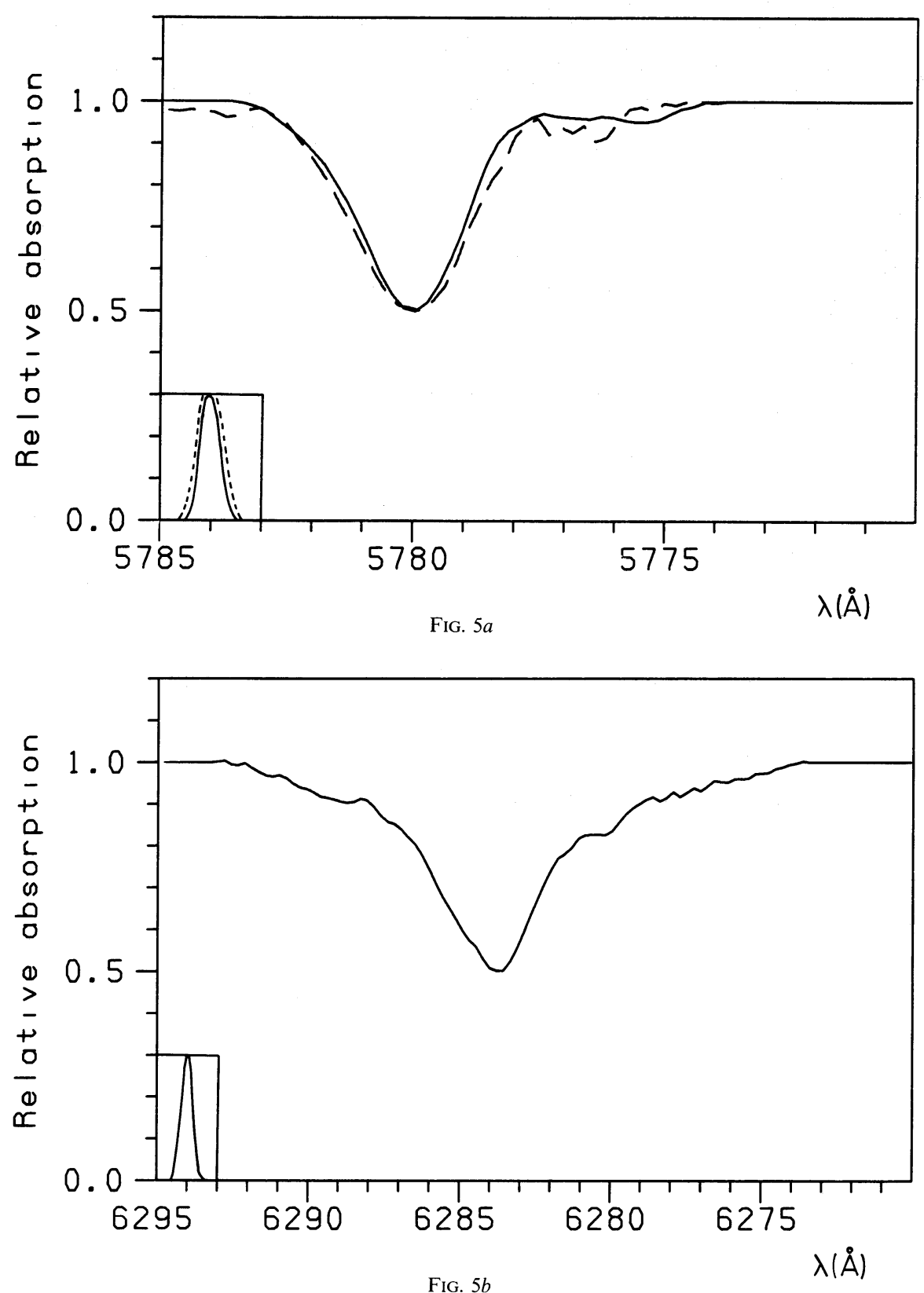

Fig. 5.-Profiles of various diffuse features normalized arbitrarily to a peak absorption of 0.5. Instrumental profile shown in lower left-hand corner includes a convolution of a rectangular point-spread function which accounts for the entrance slit, a Gaussian corresponding to a typical focusing inaccuracy of $\sim 0.2 \AA$ on the detector, and a profile due to discrete pixels on the CCD. (a) 25780 . Solid line, the average of six spectra in Cyg OB2; dashed line, the average of four stars in Be 87 . (b) 2.6284 averaged for six spectra in Cyg OB2. (c) 25797 . Solid line, the average of three highest quality spectra in Cyg OB2; dashed line, Cyg OB2 No. 5; dash-dot, Cyg OB2 No. 10. (d) $\lambda 6269$ averaged for six spectra in Cyg OB2. (e) $\lambda 6203$ and $\lambda 6205$ averaged for four spectra in Cyg OB2.

mental effect due to the lower resolution in Be 87 spectra, as can be seen from the comparison of instrumental profiles in Figure $5 a$.

The profile of $\lambda 6284$ (Fig. $5 b$ ) is strikingly different from that of 25780 . We have presented it only for the stars in Cyg OB2 since the details in Be 87 spectra are obscured by residual $\mathrm{O}_{2}$ absorption. It is important to note that the profile of $\lambda 6284$ resembles that of $\lambda 4430$, which is also characterized by little asymmetry and very broad wings (Herbig 1966). The significance of the $\lambda 6284$ profile is discussed more extensively in the following section.
Figure $5 c$ presents the profile of $\lambda 5797$ calculated from the best three spectra in Cyg OB2, without binning, i.e., with the resolution of $0.35 \AA$. The distortion of that relatively narrow line ( $1.2 \AA$ at half depth) by the instrumental profile is obvious, although the asymmetry and the general similarity to the 5780 $\AA$ profile are apparent. We have also shown the $\lambda 5797$ profile for two individual stars, Cyg OB2 No. 10 and No. 5. Since the continuum overlies both the $5797 \AA$ feature and the broad line at $5795 \AA$, the normalization of the profile to $A_{C}=0.5$ at the peak of $\lambda 5797$ can provide a measure of the relative strength of the two lines. In spite of a high noise level in the profile of 

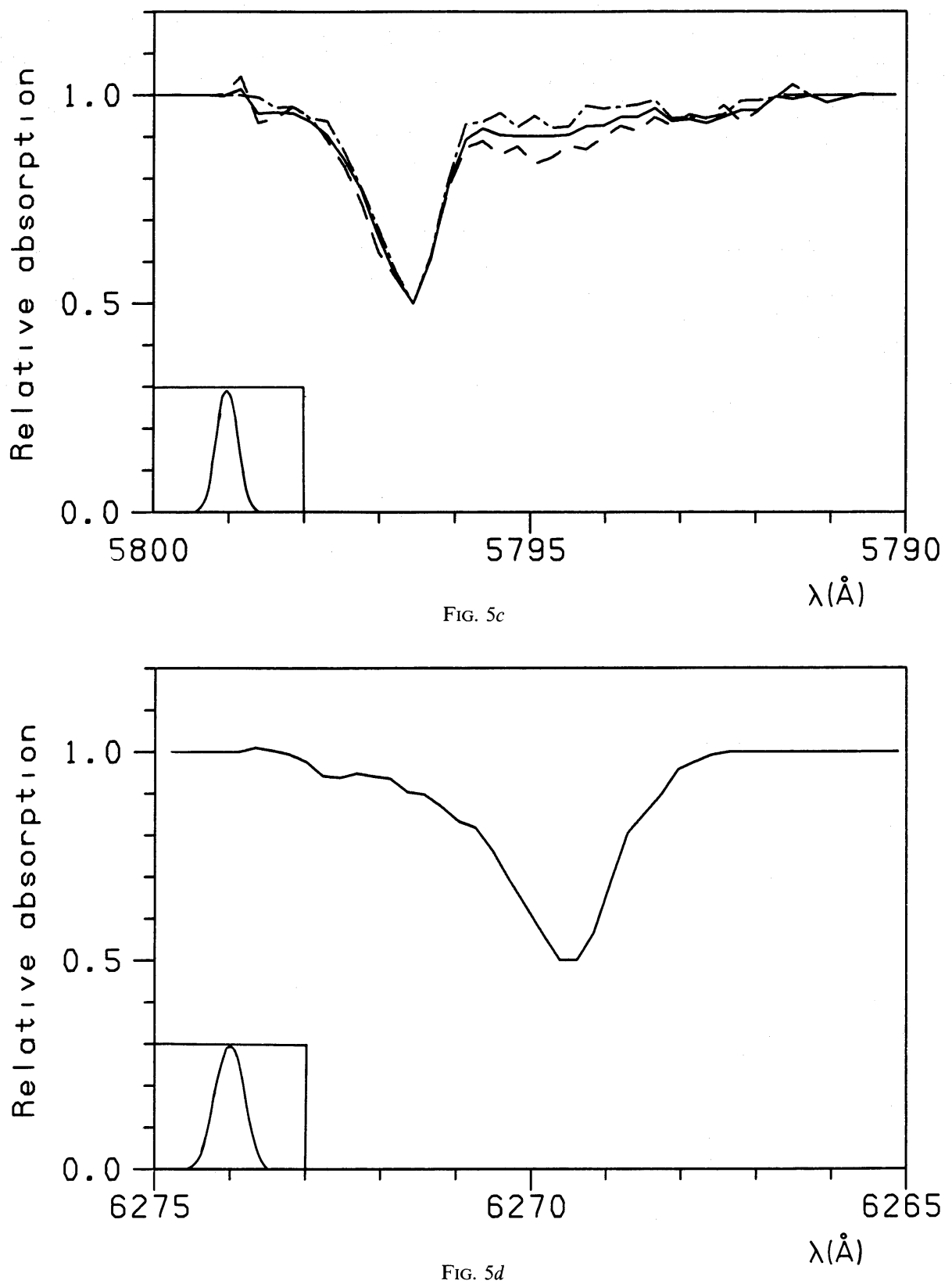

$\lambda 5795$, Figure $5 c$ suggests rather convincingly that the line is stronger relative to $\lambda 5797$ in No. 10 than in No. 5. This implies that in spite of their proximity, $\lambda 5797$ and $\lambda 5795$ may not be physically related.

The average profile of $\lambda 6269$ derived from the best four spectra in Cyg OB2 (Fig. 5d) suggests that the broadness of the wings of the line is not a result of blending. In the survey of diffuse lines by Herbig (1975), the possibility of blending is mentioned, although the line is listed as single. If this conclusion is true, $\lambda 6269$ is the most asymmetric of all single profiles observed in this program.

The profile of $\lambda 6203+\lambda 6205$ (Fig. 5e) reveals a highly complex structure involving several lines. The sudden change in the slope of the red wing of the line suggests that it consists of at least two absorption features, although the structure cannot be decomposed successfully into two Gaussians. The core of the line at $6203 \AA$ is asymmetric, with a steeper wing in the red, contrary to what is observed in other lines. If the central peak is due to a symmetric feature, the underlying broader line must be strongly asymmetric, possibly with a multiple structure. The $\lambda 6203+\lambda 6205$ feature is followed by a very shallow absorption centered at $\sim 6212 \AA$, which has escaped detection in earlier observations; it is seen clearly in all stars in Cyg OB2 and in the high S/N spectrum of Be 87 No. 4. The study of this complex series of lines in five spectra with the highest $\mathrm{S} / \mathrm{N}$ indicates that all individual features are well correlated with each other.

The fact that, at a resolution of $0.35 \AA$, no Doppler structure is apparent in the narrowest line we have observed, $\lambda 6195$, in either Cyg OB2 or Be 87, implies that Doppler effects are insignificant in the much broader profiles shown in Figure 5. The main uncertainty in the interpretation of both the correla- 


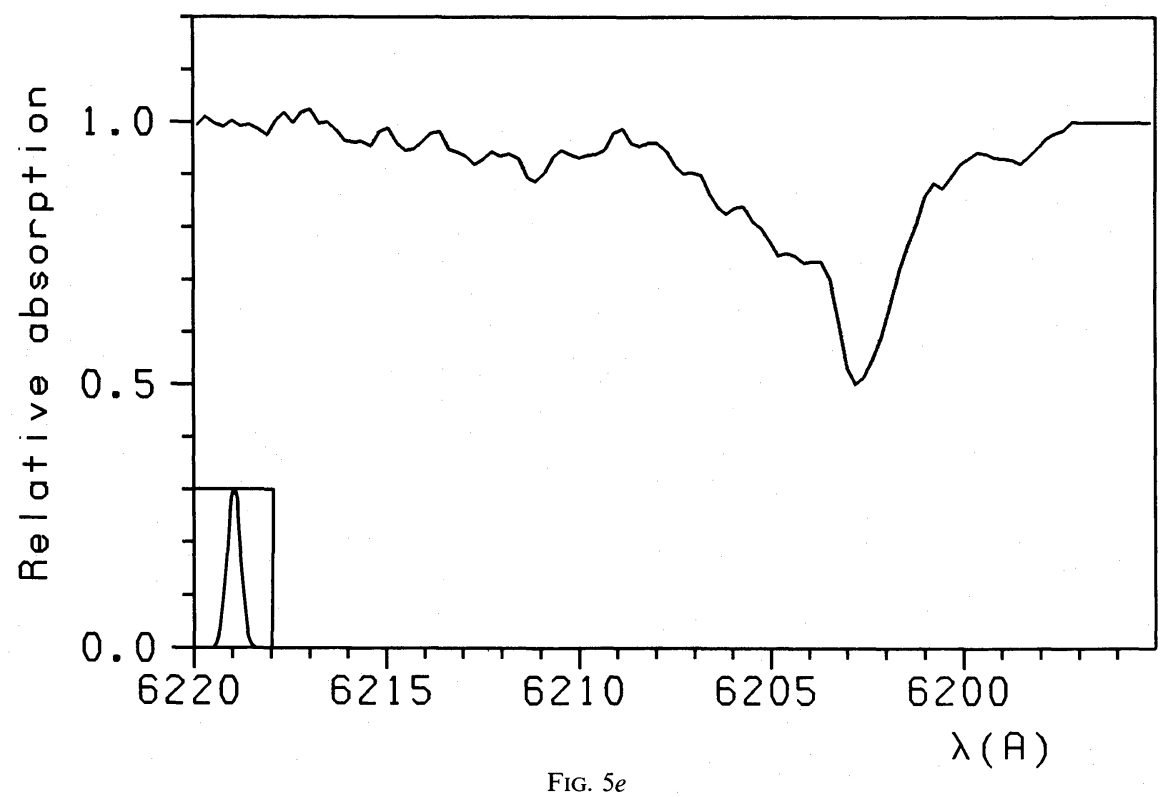

tion pattern and the profile revealed by our observations is associated with the conditions in clouds in the vicinity of Cyg OB2 and Be 87. In particular, it is not clear to what extent the correlation we have derived apply to the general diffuse medium, although the study by Wu et al. (1981) indicates that the pattern is similar, at least for $\lambda 5780$. Future observations in this series will include a full variety of cloud conditions ranging from the diffuse medium to dense regions of recent star formation.

\section{DISCUSSION}

\section{a) Column Density of Absorbers}

The number of absorbers required to produce the observed intensities of the lines provides a basic constraint on all theoretical interpretations. For optically thin lines, the column density of absorbers can be derived from the well-known formula:

$$
W_{\lambda}=\lambda^{2}\left(\frac{\pi e^{2}}{m_{e} c^{2}}\right) N_{a} f,
$$

where $W_{\lambda}$ is the equivalent width, $\lambda$ represents the central wavelength of the transition, $e^{2} / m_{e} c^{2}$ is the classical radius of the electron, $f$ denotes the oscillator strength, and $N_{a}$ is the column density of absorbers (Spitzer 1978).

The number of grains necessary to give rise to the visible extinction in the same line of sight is obtained from

$$
A_{V}=1.086 N_{\mathrm{gr}} C_{\mathrm{ext}},
$$

where $A_{V}$ is the visual extinction, $C_{\text {ext }}$ is the average extinction cross section per particle and $N_{\mathrm{gr}}$ is the coiumn density of grains.

Combining equations (1) and (2), we obtain

$$
\begin{aligned}
\frac{N_{a} f}{N_{\mathrm{gr}}} & =\frac{W_{\lambda}}{\lambda^{2} A_{V}}\left[1.086\left(\frac{\pi e^{2}}{m_{e} c^{2}}\right)^{-1} C_{\mathrm{ext}}\right] \\
& =8.324 \times 10^{7} \frac{W_{\lambda}(\mathrm{m} \AA)}{\lambda^{2}(\AA) \times A_{V}(\mathrm{mag})} .
\end{aligned}
$$

We have assumed in equation (3) that the effective cross section of visual particles is well represented by $C_{\text {ext }}=Q_{\text {ext }} \pi a^{2}$ with $a=0.12 \mu \mathrm{m}$ and $Q_{\text {ext }}=1.5$ (Greenberg 1974). The numbers derived from equation (3) can easily be expressed as $N_{a} / N_{\mathrm{H}}$ since, assuming the average gas-to-dust ratio, $N_{\mathrm{gr}}$ is of the order of $N_{\mathrm{H}} \times 10^{-12}$.

Although some of the diffuse bands in our observations are not optically thin, the use of equation (3) gives rise to errors of no more than a factor of 2 . Typically, as for $\lambda 5780$, equation (3) yields a mean value:

$$
\frac{N_{a} f}{N_{\mathrm{gr}}} \approx 5 \times 10^{2},
$$

which, for an oscillator strength $f=0.1$, implies $\sim 5000$ absorbers per grain.

\section{b) Impurity Absorption in Grains}

Theories which associate diffuse lines with grains of interstellar dust are based on the assumption that the lines are due to resonance absorptions in impurity atoms or molecules embedded in grains. In most studies, the change in the bulk optical constants of the particle has been derived from the Clausius-Mosotti equation:

$$
\frac{\epsilon-1}{\epsilon+2}=\frac{\epsilon_{0}-1}{\epsilon_{0}+2}+n \frac{4 \pi}{3} \frac{e^{2}}{m_{e}} f \frac{1}{\omega_{0}^{2}-\omega^{2}+i \gamma \omega},
$$

where $\epsilon=\epsilon^{\prime}-i \epsilon^{\prime \prime}$ is the complex dielectric constant, $\epsilon_{0}$ is the dielectric constant for the bulk material without absorbers, and $\omega_{0}$ and $\gamma$ are the peak frequency and the damping constant.

The Clausius-Mosotti equation is derived with the assumption that the local electric field acting on the absorbers is only due to the polarization induced in the dielectric by the external field. Purcell and Shapiro (1977) developed a theory which includes the correction to the local field due to the polarization induced in the dielectric by the absorber itself. Their "embedded cavity" model leads to the following formula for the modified dielectric constant.

$$
\epsilon=\epsilon_{0}+4 \pi n \alpha_{C} \frac{3 \epsilon_{0}}{2 \epsilon_{0}+1} /\left[1-\frac{\alpha_{C}}{d^{3}}\left(\frac{2 \epsilon_{0}-2}{2 \epsilon_{0}+1}\right)\right],
$$


where $d$ is the size of the spherical cavity occupied by the absorber and $\alpha_{C}$ is its total polarizability. Both theories include the assumption that the absorption resonance can be attributed to a single harmonically bound electron and use the classical approach to derive the resulting polarizability:

$$
\alpha_{C}=f \frac{e^{2}}{m_{e}} \frac{1}{\omega_{0}^{2}-\omega^{2}+i \gamma \omega} .
$$

The two models become identical for a homogeneous dielectric; i.e., when the impurity site has identical properties with normal lattice sites. For actual impurities with concentrations and lattice spacings normally encountered in solid materials, the Purcell-Shapiro model seems to provide a better description of their properties. It predicts many of the effects often observed in matrix isolation spectroscopy, such as large shifts in the peak wavelength of absorption and profile variations dependent on the type of lattice in which the absorbers are embedded (Meyer 1971).

Extensive calculations based on the Clausius-Mosotti equation have been published for various types of grains (Greenberg 1968; Wickramasinghe and Nandy 1970; Bromage 1972a, b; Greenberg and Stoeckly 1971; Greenberg and Hong 1976; Savage 1976) and the results of "embedded cavity" calculations for very small particles (corresponding to the Rayleigh approximation) have been given by Purcell and Shapiro (1977). We have supplemented the existing data with calculations based on the Purcell-Shapiro model for grain sizes in the range $0.01-0.15 \mu \mathrm{m}$ carried out for several types of grain materials (silicates and graphite with and without coatings, mantles composed of dirty ice or organic refractory). In the following we summarize the most important results.

The presence of diffuse band carriers in large grains which are responsible for the visible extinction $(a=0.12 \mu \mathrm{m})$ is excluded by the strong asymmetry predicted for such large particles. Although the embedded cavity model predicts intrinsic profile asymmetry which partly offsets the size-dependent effects of dispersion, it is not sufficient to yield a relatively symmetric outline such as that observed for $\lambda 5780$.

Very small particles $(a=0.01 \mu \mathrm{m})$ whose existence is required by the observations of UV extinction (Greenberg and Chlewicki 1983) are also excluded as impurity carriers. The dispersion effect for those particles is very small because of their low scattering efficiency, and the Clausius-Mosotti model produces an almost symmetrical profile, whereas the PurcellShapiro approach yields an asymmetric outline with a steeper wing on the red side of the line, contrary to what is observed. Further evidence that the diffuse bands are not associated with small particles comes from statistical studies which show that the bands correlate poorly with the $2200 \AA$ hump and the FUV (far ultraviolet) rise in extinction (Wu et al. 1981; Witt, Bohlin, and Stecher 1983; Nandy, Morgan, and Houziaux 1982; Seab and Snow 1984), both of which must be attributed to the $0.01 \mu \mathrm{m}$ particles in the interstellar population (Greenberg and Chlewicki 1983).

Insofar as particles are concerned, the only remaining sizes to be considered as potential diffuse line carriers are in the intermediate range $(0.02-0.10 \mu \mathrm{m})$. The typical size of "diffuse line" particles indicated by the observed profiles (Fig. 5) is $\sim 0.05 \mu \mathrm{m}$. However, using absorbers embedded in intermediate-size particles, it is difficult to explain both the details of individual profiles, such as the lack of broad wings in 25780, and the large variety of observed line shapes. In our calculations, which included several grain materials and a full range of physically acceptable cavity parameters, we could not reproduce the observed range of asymmetry with absorbers embedded in a single size particle. Consequently, the impurity absorption mechanism requires that various diffuse lines should be produced by particles of different sizes and impurity contents. This requirement is not only inconsistent with the present picture of interstellar grains and their formation, but also appears to contradict the observed correlation between such lines as $\lambda 5780$ and $\lambda 6284$.

Perhaps the greatest difficulty in grain impurity theories is the observed invariability of the peak wavelengths of the lines. This seems to require that identical impurity sites should be present in all interstellar particles, since the wavelength of impurity absorption depends strongly on the coupling with the lattice. However, the large variety of conditions under which grains are formed and subsequently processed in diffuse and molecular clouds makes it much more likely that, although the basic chemical composition of the particles is uniform in the interstellar medium, there should be a wide range of impurity site parameters. The peak wavelength of absorption is also known to vary strongly as a function of the temperature of the lattice, but the observations of the lines in regions where grains are hotter than in the diffuse medium (the Orion Nebula; Baines and Whittet 1983) have shown no shifts above the likely Doppler effects of $\sim 1 \AA$.

The evolution of the separate population of intermediate size particles containing diffuse band carriers poses another difficulty for the solid state theory of the lines. Since the intensity of the lines relative to $E_{B-V}$ varies by no more than a factor of $\sim 2$ between different lines of sight, the total amount of material contained in the "diffuse band" population relative to the population of grains responsible for the visible extinction should remain approximately constant (within a factor of the order of 1) over the typical lifetime of an interstellar particle $\left(\sim 10^{9} \mathrm{yr}\right)$. Diffuse band particles, whose sizes must exceed $\sim 0.02 \mu \mathrm{m}$, would not be prevented by temperature fluctuations from accreting condensable material in molecular clouds. If all the accreted material is quickly lost when the particles emerge into the diffuse medium, the remaining core will be exposed to rapid erosion, which destroys basic materials, such as graphite or silicates, $\sim 10$ times faster than the rate of their replenishment (Draine and Salpeter 1979a,b). On the other hand, if the particles retain a polymerized refractory mantle after entering the diffuse medium, each passage through a molecular cloud will result in a permanent increase in their size (Greenberg 1982; Greenberg 1985). The relative growth rate in molecular clouds, expressed as $\left[(d a / d t) \times a^{-1}\right]$, is a steep function of size: since all the growth processes are roughly proportional to the cross section, the growth rate should approximately vary like $a^{-1}$ (Greenberg 1978). The destruction rate in the diffuse medium, determined predominantly by sputtering, is only a very slowly varying function of size (Draine and Salpeter 1979b). The stability of visible extinction curves in the diffuse medium indicates that the growth and destruction rates are closely balanced for the large particles $(a \approx 0.125 \mu \mathrm{m})$ which provide most of the extinction at visible wavelengths. For the diffuse band population, with a typical particle size of $0.05 \mu \mathrm{m}$, the relative growth is higher by a factor of 2 , whereas the destruction rate remains approximately the same. This would result in an increase of mass contained in the diffuse band population, with respect to larger grains, by a factor of $\sim 2$ over each cycle of passing between molecular and diffuse 
clouds. Since there are $\sim 50$ such cycles in the lifetime of a particle, the rapid growth of smaller grains should lead to a much larger variability of diffuse band intensities relative $E_{B-V}$ than the observed value of $\sim 2$.

In view of all the objections we have outlined, the impurity model appears highly unlikely because the special particle properties it requires are apparently inconsistent with observational constraints on interstellar grains. In the following section, we shall demonstrate that most of the difficulties can be overcome by the alternative, molecular explanation.

\section{c) Absorptions in Gas-Phase Molecules}

Models alternative to grain impurity theories associate the origin of the diffuse lines with molecules present in the gas phase of the interstellar medium. The observed width of the lines $\left(1-100 \mathrm{~cm}^{-1}\right)$ can be explained by unresolved rotational structures (Danks and Lambert 1976; Ross 1971; Hollas 1973), predissociation (Herzberg 1955), or changes in the lifetime of excited states due to internal energy conversion (Robinson 1967; Jortner, Rice, and Hochstrasser 1969; Smith 1971). A key requirement in the molecular hypothesis is the stability of the species against photodissociation by the interstellar radiation field, which favors certain types of large molecules. The large molecule concept has been considered in the past by several authors. A variety of organic molecules have been proposed as possible carriers of diffuse features, such as polycyclic molecules (Donn 1968; Johnson 1967, and discussion after paper; Johnson 1972) and linear carbon chains (Douglas 1977). Fresh interest in molecular theories of diffuse lines has been aroused by the suggestion that polycyclic aromatic hydrocarbons may account for some of the diffuse bands (Legér and d'Hendecourt 1985; van der Zwet and Allamandola 1985).

The general characteristics of the diffuse bands so far observed are consistent with the properties of large molecules, and some of the properties established in this study strongly favor the large molecule explanation. Complex profiles such as the $\lambda 6203+\lambda 6205+\lambda 6212$ structure are reminiscent of line shapes produced by unresolved rotational envelopes in vibronic transition of large molecules (Ross 1971; Hollas 1973). The profiles of most of the relatively narrow diffuse lines (Herbig's class A), such as $\lambda 5780$ or $\lambda 5797$, are easily accounted for as molecular absorptions, whereas the lack of broad Lorentzian wings poses a severe difficulty in solid state theories; van der Zwet and Allamandola (1985) have shown that the asymmetry of the lines is consistent with rotational excitations at typical temperatures in the diffuse interstellar medium $(\sim 100 \mathrm{~K})$. The two broadest and strongest class A features, $\lambda 6284$ (Fig. 5b) and $\lambda 4430$ (Herbig 1966) have nearly symmetrical Lorentzian profiles, very different from those of narrower lines. The widths of these features can be explained by internal energy conversion (Douglas 1977; Van der Zwet and Allamandola 1985); the required lifetimes are $\sim 10^{-13} \mathrm{~s}$ and are consistent with spectroscopic data for large molecules (Byrne and Ross 1971). It is natural to expect that Lorentzian profiles due to lifetime broadening should appear only in lines whose widths exceeds the temperature dependent effects of rotational excitations, which tend to produce asymmetric shapes. There is no equally natural explanation for the occurrence of symmetric Lorentzian wings only in the broadest lines in solid state theories, unless one arbitrarily assumes that the broadest features are due to carriers embedded in smaller particles.

Spectroscopic considerations indicate that visible absorptions, particularly at wavelengths as long as 6000-7000 $\AA$, are likely to be due to ionized rather than neutral molecules.
Approximate calculations indicate that for many large molecules there could exist similar amounts of ionized and neutral species in the interstellar medium (Legér and d'Hendecourt 1985; van der Zwet and Allamandola 1985). Differences in the UV radiation field could therefore result in large relative changes in the number of ionized molecules, which would give rise to variations in line intensities with respect to reddening, such as those observed for lines in group II. At the same time, the wide range of properties expected even for a single generic group of molecules, such as polycyclic aromatic hydrocarbons (e.g., large differences in ionization potentials or recombination coefficients), allows the possibility that certain species could be either predominantly neutral or mainly ionized, giving rise to lines such as $\lambda 5797$ which correlate strongly with $E_{B-V}$. Interpreted within the framework of the spectroscopy of large molecules, the observed properties of the diffuse bands argue for a small number of species (however, certainly more than one, as shown by different correlations), presumably with common generic characteristics.

The molecular explanation of the origin of diffuse features may also involve very small ( $\lesssim 0.001 \mu \mathrm{m})$ amorphous particles rather than large molecules. The properties of such particles have certain "molecular" aspects; namely, their internal energy is not thermalized and is predominantly contained in several discrete vibrational modes. On the other hand, a relatively wide spectrum of combination modes may resemble the continuum phonon distribution of bulk solids. Since the properties of large molecules can be described in similar terms, the distinction between $10 \AA$ amorphous particles and molecules cannot be applied at this stage when no spectroscopic identifications are available.

\section{CONCLUSIONS}

We have observed the diffuse interstellar bands in early-type stars in three OB associations with the extinction attributed mostly to material at intermediate densities between molecular clouds and the diffuse medium. The degree of correlation with $E_{B-V}$ separates the lines into at least two groups. The analysis of line profiles, which was carried out in much greater detail than had previously been possible, reveals a wide variety of shapes even among strongly correlated lines, ranging from a nearly symmetric Lorentzian for $\lambda 6284$ to a highly complex multiple structure of $\lambda 6203+\lambda 6205$. These observational facts appear to preclude the origin of the lines in bulk solid particles and favor their explanation as vibronic transitions in large molecules.

We are very grateful to $\mathrm{R}$. Terlevich, whose invaluable help and advice as support astronomer of our observing project have made its successful realization possible. We are also indebted to $R$. le Poole for valuable discussions on many aspects of this paper and to L. d'Hendecourt for stimulating conversations about recent theoretical developments. G. C. and P. P. A. would like to thank the RGO staff at the Roque de los Muchachos Observatory for the hospitality and efficiency they have experienced during their stay as guest observers.

G. P. v. d. Z. and G. C. gratefully acknowledge the support of the Stichting voor Fundamenteel Onderzoek der Materie (F.O.M.) and the Kerkhoven-Boscha Foundation, respectively.

The Isaac Newton telescope, on the island of La Palma, is operated by the Royal Greenwich Observatory at the Spanish Observatorio del Roque de los Muchachos of the Instituto de Astrofisica de Canarias. 
Abbott, D. C., Bieging, J. H., and Churchwell, E. 1981, Ap. J., 250, 645.

Baines, D. W. T., and Whittet, D. C. B. 1983, M.N.R.A.S., 203, 419.

Bohannan, B., and Conti, P. S. 1976, Ap. J., 204, 797.

Bromage, G. E. $1972 a$, Ap. Space Sci., 15, 426.

. 1972b, Ap. Space Sci., 18, 449.

Bromage, G. E., and Nandy, K. 1973, Astr. Ap., 26, 17.

Byrne, J. P., and Ross, I. G. 1971, Australian J. Chem., 24, 1107.

Chlewicki, G., Greenberg, J. M., Aiello, S., Barsella, B., Patriarchi, P., and

Perionotto, M. 1984, in Proc. 4th European IUE Conf. (ESA SP-218), p. 507.

Danks, A. C., and Lambert, D. L. 1976, M.N.R.A.S., 174, 571.

Donn, B. 1968, Ap. J. (Letters), 152, L129.

Douglas, A. E. 1977, Nature, 269, 130.

Draine, B. T., and Salpeter, E. E. $1979 a, A p$. J., 230, 106. $1979 b$, Ap. J., 231, 438.

Eiroa, C., Elsasser, M., and Lahulla, J. F. 1978, Astr. Ap., 74, 89.

Greenberg, J. M. 1968, in Stars and Stellar Systems, Vol. 7, Nebulae and Interstellar Matter, ed. B. M. Middlehurst and L. H. Aller (Chicago: University of Chicago Press), p. 221.

. 1974, Ap. J.(Letters), 188, L81.

. 1978, in Cosmic Dust, ed. J. A. M. McDonnell (New York: Wiley), p. 187

1982, in Submillimetre Wave Astronomy, ed. J. E. Beckman and J. P.

Phillips (Cambridge: Cambridge University Press), p. 261.

1985, in Birth and Infancy of Stars, ed. R. Lucas, A. Omont, and R.

Stora (Amsterdam: North-Holland), p. 138.

Greenberg, J. M., and Chlewicki, G. 1983, Ap. J., 272, 563.

Greenberg, J. M., and Hong, S.-S. 1976, Ap. Space Sci., 39, 31

Greenberg, J. M., and Stoeckly, R. 1971, Nature Phys. Sci., 230, 15.

Hall, D. S. 1974, Acta Astr., 24, 69.

Herbig, G. H. 1966, Zs. Ap., 64, 512. 1975, Ap.J., 196, 129.

Herzberg, G. 1955, Mém. Soc. Roy. Sci. Liège, 15, 291.

Hollas, J. M. 1973, J. Chem. Phys., 1, 63.

Johnson, F. M. 1967, in Interstellar Grains, ed. J. M. Greenberg and T. P. Roarck (NASA/SP-140), p. 229.

Johnson, F. M. 1972, Mém. Soc. Roy. Sci. Liège, 6e sér. 3, 391.

\section{REFERENCES}

Johnson, H. L. 1968, in Stars and Stellar Systems, Vol. 7, Nebulae and Interstellar Matter, ed. B. M. Middlehurst and L. H. Aller (Chicago: University of Chicago Press), p. 167.

Jortner, J., Rice, S. A., and Hochstrasser, R. M. 1969, Adv. Photochem., 7, 149.

Lada, C. J., Elmegreen, B. G., Cong, H. J., and Thaddeus, P. 1978, Ap. J. (Letters), 226, L39.

Leger, A., and d'Hendecourt, L. B. 1985, Astr. Ap., 146, 81

Merrill, P. W., 1934, Pub. A.S.P., 46, 206.

Meyer, B. 1971, Low Temperature Spectroscopy (New York: Elsevier)

Morgan, W. M., Johnson, H. L., and Roman, N. G. 1954, Pub. A.S.P., 66, 85.

Nandy, K., Morgan, D. H., and Houziaux, L. 1982, Ap. Space Sci., 85, 221.

Pitault, A. 1981, Astr. Ap., 97, L5.

Purcell, B. M., and Shapiro, P. R. 1977, Ap. J, 214, 92

Robinson, G. W. 1967, J. Chem. Phys., 47, 1967.

Ross, I. G. 1971, Adv. Chem. Phys., 20, 341.

Savage, B. D. 1976, Ap. J., 205, 122

Schulte, D. H. $1958, A p . J .128,41$.

Seab, C. G., and Snow, T. P. 1984, Ap. J. 277, 200.

Smith, W. H. 1971, J. Chem. Phys., 54, 4169.

Snell, R. L., and Vanden Bout, P. A. 1981, Ap. J., 244, 844.

Snow, T. P., and Cohen, J. G. 1974, Ap. J., 194, 313

Snow, T. P., York, D. G., and Welty, D. E. 1977, A.J., 82, 113.

Souza, S. P., and Lutz, B. L. $1980 a$, Ap. J. (Letters), 235, L87.

. 1980b, in IAU Symposium 87, Interstellar Molecules, ed. B. H. Andrew (Dordrecht: Reidel), p. 261.

Spitzer, L., Jr. 1978, Physical Processes in the Interstellar Medium (New York: John Wiley).

Turner, D. G., and Forbes, D. 1982, Pub. A.S.P., 94, 789.

van der Zwet, G. P., and Allamandola, L. J. 1985, Astr. Ap., 146, 76.

Voelcker, K., and Elsasser, H. 1973, in IAU Symposium 52, Interstellar Dust and Related Topics, ed. J. M. Greenberg and H. C. van der Hulst (Dordrecht: Boston), p. 529.

Wickramasinghe, N. C., and Nandy, K. 1970, Ap. Space Sci., 6, 154

Witt, A. N., Bohlin, R. C., and Stecher, T. P. 1983, Ap. J. (Letters), 267, L47.

Wu, C.-C. 1972, Ap. J., 178, 681.

Wu, C.-C., York, D. G., and Snow, T. P. 1981, A.J., 86, 755.

P. P. Alvarez: Instituto de Astrofisica de Canarias, Santa Cruz de Tenerife, Spain

G. CHLEWICKI: Laboratory for Space Research, Postbus 800, NL-9700 AV, Groningen, The Netherlands

J. Mayo Greenberg, G. P. Van der Zwet, and L. J. Van Ijzendoorn: Astrophysics Laboratory, Huygens Laboratory, Wassenaarseweg 78, 2300 RA Leiden, Holland 